\title{
A Wearable Carotid Ultrasound Assembly for Early Detection of Cardiovascular Diseases
}

\author{
Sumaiya Shomaji*1, Abhishek Basak ${ }^{2}$, Soumyajit Mandal ${ }^{2}$, Robert Karam ${ }^{1}$ and Swarup Bhunia ${ }^{1}$ \\ Email: shomaji@ufl.edu \\ ${ }^{1}$ Dept. of ECE, University of Florida, Gainesville, FL 32608. \\ ${ }^{2}$ Dept. of EECS, Case Western Reserve University, Cleveland, OH 44106
}

\begin{abstract}
Cardiovascular disease is currently responsible for a major portion of all global deaths. Fortunately, early detection of its symptoms can greatly contribute to effective prevention. Hence, clinicians recommend routine cardiovascular check-ups. Even though various methods for cardiac diagnosis exist, most of them are clinic-based, and therefore time-consuming and costly. Therefore, it is not always possible for patients to go through these procedures on a regular basis. In this paper, we propose a novel wearable ultrasonic imaging assembly for routine, easy-to-use, and economical monitoring of the carotid arteries. The device monitors intima-media thickness (IMT), which is a proven clinically useful marker for diagnosis of cardiovascular disease and prediction of imminent cardiovascular events. It uses standard B-mode ultrasound, which is suitable for implementation within area- and power-constrained wearable form factors. We present design parameters and power requirements for all the essential hardware components of the proposed wearable imaging system. We also present an efficient algorithm for predicting IMT anomalies from ultrasound images.
\end{abstract}

\section{INTRODUCTION}

Cardiovascular diseases (CVDs), including coronary heart diseases (CHDs) and stroke, are the leading causes of death globally. According to the World Health Organization (WHO), CVDs accounted for $31 \%$ of all global deaths in 2012, of which CHD and stroke accounted for $42 \%$ and $38 \%$, respectively [1]. Therefore, diagnosis and treatment of CVDs is imperative for reducing mortality caused by these diseases. Fortunately, a number of studies have suggested that early detection and diagnosis may significantly lower the risk of CVD-related deaths [2].

Numerous cardiac diagnosis tools are available today, including electrocardiography, stress tests, echocardiography, chest X-rays, blood tests, coronary angiography, cardiac catheterization, CT heart scans, myocardial biopsies, heart MRI, and pericardiocentesis. Typically, the required test facilities are available at clinics and hospitals. Therefore, cardiac diagnosis requires individuals to visit a suitable care facility, where the appropriate tests are performed under the supervision of either a doctor or an experienced operator. Moreover, most of these tests are expensive and timeconsuming. As a result, routine check-ups are not convenient for a large segment of at-risk population. Hence, there is a need for economical and easily-accessible solutions that enable regular cardiac monitoring by eliminating the need to visit a care facility. In this paper, we propose a novel wearable point-of-care ultrasound device that addresses this

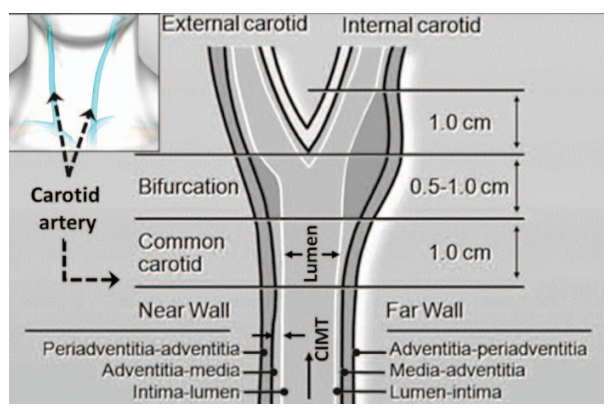

Fig. 1. Segments and typical dimensions of a single carotid artery along with its location within the human neck (adapted from [3]).

need by monitoring the carotid arteries to reliably predict the risk of future cardiovascular events.

Arteries carry oxygen-rich blood from the heart to other tissues and organs. The two carotid arteries are located on either side of the neck, as shown in Fig. 1. They are directly linked to the left ventricle (LV) through the aorta. The LV pumps oxygenated blood into the aorta, which then supplies it to upper body parts through the carotid arteries. The health of the carotid arteries is closely associated with that of the overall cardiovascular system [4]. For example, atherosclerosis - deposits of plaque on the arterial walls - is a well-known risk factor for myocardial infarction and stroke. Plaque is a waxy substance consisting of fat, cholesterol, calcium, and other components that are normally found in dissolved form within the blood. Plaque can harden with time, which causes narrowing of the corresponding artery (stenosis) and thus obstructs the flow of oxygen-rich blood. Various studies have shown that ultrasound detection of carotid stenosis can be used to predict CVDs [4], [5]. Our device relies on this principle to facilitate early detection of future cardiac events using low-cost carotid monitoring.

Carotid ultrasound is a painless, harmless, and noninvasive test that detects plaque buildup in these arteries. In this paper, we propose a novel wearable ultrasound device that uses carotid ultrasound to predict cardiovascular health. The device is shaped like a neck brace, as shown in Fig. 2. Two ultrasonic transducer array probes are placed at strategic locations on the brace in order to monitor both carotid arteries. The resulting images are then analyzed to detect abnormalities in carotid intima-media thickness (CIMT). CIMT is measured between the intima-lumen and media-adventitia interfaces, as shown in Fig. 1. Plaque formation within the 


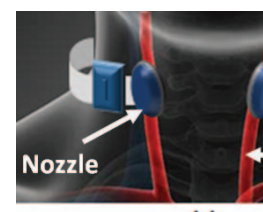

(a)

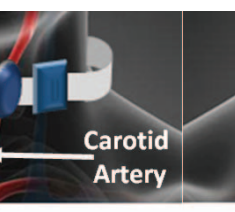

nt

Fig. 2. Placement of the proposed device during operation: (a) Nozzle placement while releasing ultrasound gel; (b) Transducer array placement on strategic locations during diagnosis.

inner artery lining causes CIMT to increase beyond the normal range observed in asymptomatic individuals. The device issues a warning when it detects abnormally large values of CIMT, and recommends that the user visit a clinic or hospital for a thorough cardiovascular check-up. In this way it provides early diagnosis of CVDs.

The proposed system has the following benefits: i) simple design and non-invasive diagnostic technique, which allows individuals to operate the device without special training; ii) autonomous routine screening and detection without the need to visit a care facility; iii) point-of-care monitoring and early detection of future CVDs, thus reducing the risk of myocardial infarction and stroke; iv) reduction of CVD treatment costs, which are borne by the government and patients and in 2011 were estimated to be $\$ 320.1$ billion in the United States alone [6]; v) wearable form-factor; and vi) low-cost and affordable design.

Several carotid artery monitoring devices have been proposed [7], [8]. These devices either use Doppler-mode ultrasound or stethoscopes to study blood flow in the carotid arteries. They also typically require large, expensive hardware, in conjunction with complex diagnostic algorithms, to be viable. In addition, very few of the studies have used the diagnosis results specifically for CVD detection [7], [8]. Other devices, which use either NMR spectroscopy or MRI for detecting carotid tissue properties as indicators of future CVD [9], [10], do not come in a low-cost, wearable form factor. Therefore, existing methods are not viable for an economical point-of-care device. Conversely, the proposed device aims to predict CVD using CIMT and lumen diameter measurements as surrogate markers, rather than measurements of blood flow. It uses B-mode ultrasound, which is a widely recognized method for CIMT measurement that can be implemented using miniaturized and low-cost instrumentation. In particular, the paper makes the following novel contributions:

1) It proposes a wearable imaging system that uses B-mode ultrasound to detect carotid stenosis and predict CVDs.

2) It identifies the key design parameters of the proposed system, and discusses how their values are chosen.

3) It presents an image processing algorithm that enables automatic detection of stenosis in the carotid arteries.

To the best of our knowledge, this is the first effort on developing a simple and cost-effective wearable ultrasound device specifically designed to predict CVDs by monitoring the carotid arteries.

\section{SySTEM DESIGN}

The proposed device will be integrated within a neckbrace-like structure, as shown in Fig. 2. Important ultrasound

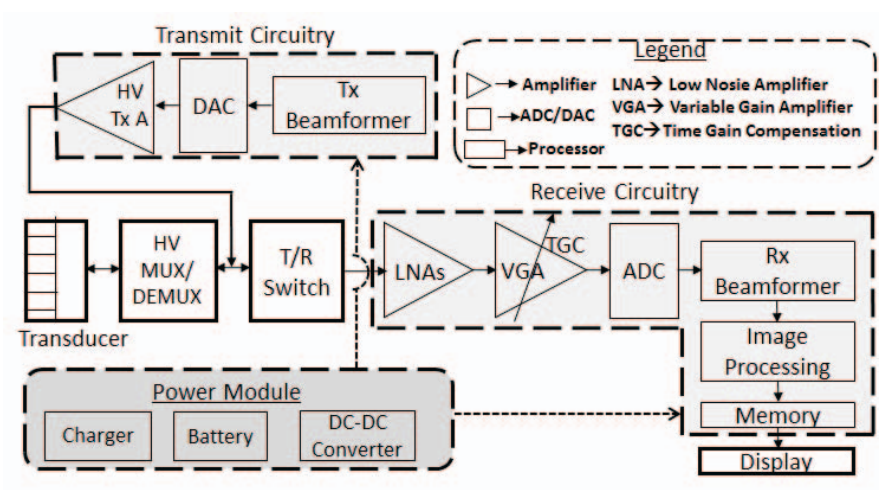

Fig. 3. System-level block diagram for the proposed carotid monitoring device showing the transducer, transmit/receive circuitry, and power module.

TABLE I

OPTIMIZED VALUES OF THE KEY DESIGN PARAMETERS.

\begin{tabular}{cc}
\hline Parameter & Value \\
\hline Transducer type & Linear array \\
Number of elements & 128 \\
Scan dimension per artery & $4 \mathrm{~cm} \times 4 \mathrm{~cm}$ \\
Transducer frequency & $7.5 \mathrm{MHz}$ \\
Element pitch & $0.3 \mathrm{~mm}$ \\
Depth of focus & $4 \mathrm{~cm}$ \\
Dynamic range & $60 \mathrm{~dB}$ \\
Optimal frame rate & $25 \mathrm{~Hz}$ \\
\hline
\end{tabular}

design parameters and their optimized values considering the design constraints are listed in Table I. The major system components are shown in Fig. 3 and described here:

1) Transducer: Since our goal is to develop a wearable device, the hardware has to be compact and operate in an energy-efficient manner while providing highquality images. Previous results suggest that linear array transducers are preferable for B-mode ultrasound of the carotid arteries [11]. Therefore, a 1-D linear array is used to create 2-D images of each artery and nearby tissue. Beam steering and dynamic focusing are useful for imaging blood flow via Doppler measurements and for scanning large areas at deep penetration depths, respectively [12]; neither is required for this system. Specifically, the depth of focus for the carotid arteries is only $\sim 4 \mathrm{~cm}$, which is small enough for a linear array to measure without any dynamic focusing. The proposed design parameters for the array are described below:

- Frequency: The transducer frequency is set at 7.5 $\mathrm{MHz}$ for the desired resolution. At a typical attenuation rate of $1 \mathrm{~dB} / \mathrm{cm} / \mathrm{MHz}$, a dynamic range of $60 \mathrm{~dB}$ easily provides an imaging depth of $4 \mathrm{~cm}$ at this frequency. The frame rate for our device should be $>15 \mathrm{~Hz}$ to ensure good image quality and to facilitate edge detection; we choose a rate of $25 \mathrm{~Hz}$.

- Element Pattern: Capacitive Micro-machined Ultrasound Transducers (CMUTs) are capable of providing better resolution compared to equivalent piezoelectric 


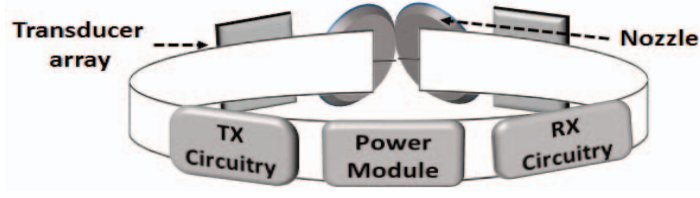

Fig. 4. Rear portion of the device showing the locations of transmit and receive circuitry along with the power module.

transducer arrays. Therefore, we use CMUTs to improve the definition of fine structures within the arteries. Specifically, a 128-element linear CMUT array will be a suitable compromise between resolution and manufacturing cost [13]. Two independent arrays are placed on the neck to simultaneously monitor both carotid arteries, as shown in Fig. 2. Array placement is discussed in the next section.

- Scan Dimension for a Single Array: Each transducer array should be capable of scanning an area of at least $4 \mathrm{~cm} \times 4 \mathrm{~cm}$ with a depth of focus of at least $4 \mathrm{~cm}$ from the body surface in order to monitor the artery, as shown in Fig. 1.

2) Transmit (Tx) Circuitry is located towards the back of the neck brace (Fig. 4). It consists of a Tx beamformer, a digital-to-analog converter (DAC), and a high voltage (HV) Tx amplifier (pulser).

3) Receive ( $\mathbf{R x})$ Circuitry is also housed towards the back (Fig. 4). It consists of a low-noise amplifier (LNA), a variable gain amplifier (VGA) to implement time gain compensation (TGC), an analog-to-digital converter (ADC), a Rx beamformer, and an image processor with display unit.

4) Transducer-Circuit Interface: A $(128 \times 1) \quad \mathrm{HV}$ mux/demux and a Tx/Rx switch are located near the Tx and Rx circuitry. The mux/demux reduces the complexity of $\mathrm{Tx}$ and $\mathrm{Rx}$ hardware at the cost of flexibility, whereas the switch protects the Rx circuitry from HV transmission pulses.

5) Power module: Supplies power to the system, and consists of a charging unit, rechargeable battery, and flow controller. This module is also placed on the back.

6) Storage: Consists of both volatile and nonvolatile memory. The former buffers raw acquired data prior to image formation and analysis, while the latter stores programs, configuration parameters, and previous measurements (for comparison and assessment purposes).

7) Nozzle: Acoustic gel is essential for efficient transmission of ultrasound energy into the body. Two electrically-controlled nozzles are present on the brace; they can be used to automatically apply gel on the focused area.

8) Strap: A strap is used for easy adjustment of the brace on the patient's neck.

The total capacitance of the array is estimated to be 203 $\mathrm{pF}$, corresponding to a per-element capacitance of $\sim 1.6 \mathrm{pF}$. The chosen excitation voltage is $30 \mathrm{~V}$, resulting in an estimated peak current of $\sim 2.3 \mathrm{~mA}$ per element at $7.5 \mathrm{MHz}$. The corresponding peak power levels are $\sim 72 \mathrm{~mW}$ and $\sim 9 \mathrm{~W}$ for individual elements and the entire array, respectively.

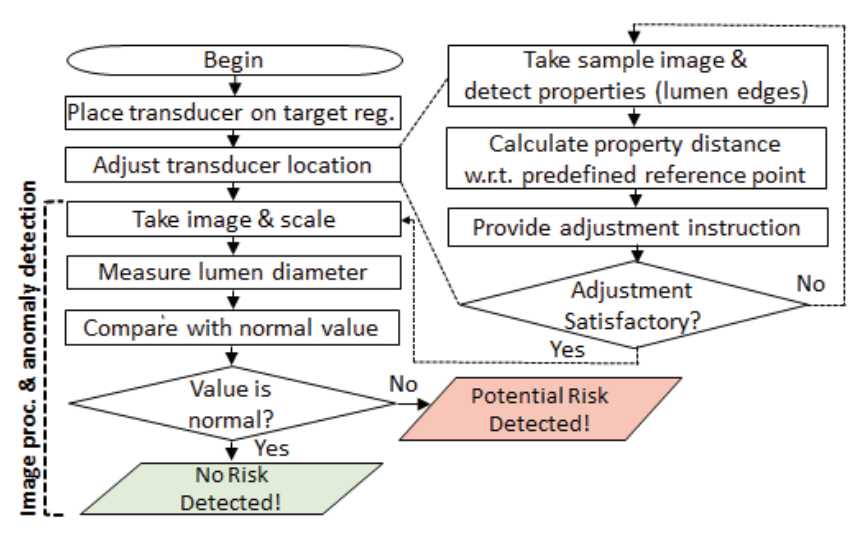

Fig. 5. Flow of operation for a single cycle of diagnosis including array placement followed by image processing for automatic anomaly detection.

\section{SYSTEM OPERATION}

The proposed device can measure the common carotid artery (CCA), carotid bifurcation (BIF), internal carotid artery (ICA), and the mean or maximal IMT. These structures have similar predictive power for future CVDs [4], [5], but a majority of prior studies have considered the CCA or ICA [14]. For better results, one can consider scanning from three angles: (a) anterior, (b) lateral, and (c) posterior. The brace should be placed on these locations successively.

Operation: The proposed device is configured as a neck brace, as described earlier. Two flexible transducer arrays will be located in the front along with the nozzles, and the Tx/Rx circuitry and power modules will be located towards the rear. Subjects must remain as still as possible during operation.

1) First, the brace should be worn while keeping the nozzles in contact with the skin, as shown in Fig. 2(a). The nozzles release gel when pressed, thus making the imaged regions ready for efficient ultrasound transmission. They are then moved aside to create space for the transducer arrays.

2) Correct placement of transducers is an important prerequisite for successful diagnosis. Necessary measurements are done and compared with predefined ideal data to ensure proper transducer placement. To illustrate, the transducers are placed in strategic locations (Fig. 2(b)) following the algorithm shown in Fig. 5. A sample image is taken on which the previously fixed reference point is searched. The point normally tends to be located in the middle of the lumen diameter line. As soon as the point is traced, relative distance is measured between the near and far wall lumen edges to the searched point. If both distances are equal or very close, the device registers that the transducers are placed correctly. If not, the device will indicate to the subject how to move the transducers. The device will be ready to take the final ultrasound image once an acceptable location has been reached.

3) The device processes the acquired image to extract both CIMT and lumen diameter. A variety of existing algorithms can be incorporated into the device to obtain these measurements [15]. However, we propose a novel lumen-diameterbased anomaly detection algorithm (Fig. 5). When plaque forms, CIMT tends to increase, thus causing the lumen 


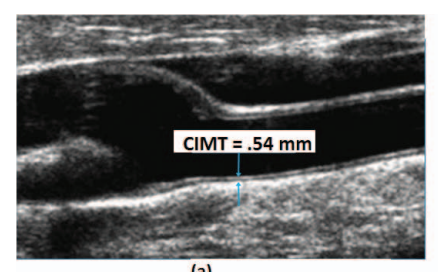

(a)

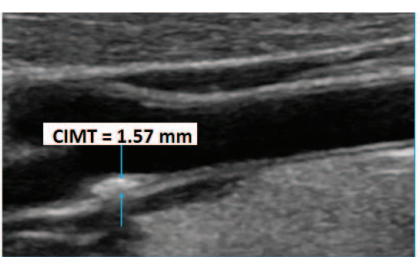

(b)

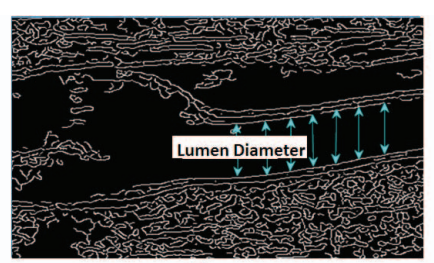

(c)

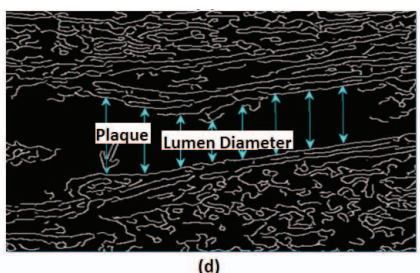

(d)

Fig. 6. Raw images processed for lumen diameter measurement: (a) raw image without plaque; (b) raw image with plaque; (c) processed version of (a) for lumen diameter measurement; and (d) processed version of (b) for lumen diameter measurement.

diameter to decrease. The proposed algorithm compares the measured lumen diameter (distance between the near and far wall lumen edges) with the normal range. The acceptable ranges of CIMT and lumen diameter are $0.4 \mathrm{~mm}$ to $0.8 \mathrm{~mm}$ and $4.3 \mathrm{~mm}$ to $7.7 \mathrm{~mm}$, respectively [16]. Anomalous values indicate a potential problem, which is indicated to the user.

Results may vary due to several effects during operation; a few guidelines should be followed to ensure better results. Firstly, the user should remove all clothing and jewelry in the area to be examined. Hence a loose-fitting, open necked shirt or blouse is ideal. Secondly, the user should lie on his/her back during the examination process. Finally, ultrasound examinations are very sensitive to motion, so activities during the examination can prolong the process. Therefore, it is better for the user to be at rest during the examination.

\section{RESUlTS}

The proposed algorithm was evaluated using several raw ultrasound test images [17] which are representative of those taken by the proposed wearable device. For each image, the lumen diameter was automatically measured within the CCA segment. Processing Fig. 6(a) yields Fig. 6(c), with a measured lumen diameter of $5.31 \mathrm{~mm}$, which is within the normal range. Processing Fig. 6(b) yields Fig. 6(d), with a lumen diameter of $1.57 \mathrm{~mm}$, which is significantly below the normal range, indicating an anomaly. These results agree well with the manually measured values for CIMT, which indicate Fig. 6(a) is within the normal range $(0.54 \mathrm{~mm})$, while Fig. 6(b) is well above normal $(1.57 \mathrm{~mm})$. Therefore, both automated and manual analysis can detect the narrowing of the CCA in Fig. 6(b).

\section{CONCLusion}

We have presented a novel wearable ultrasound imaging device for carotid artery monitoring. We have discussed the system design requirements. We have also presented a case study showing promising preliminary results using an autonomous framework for anomaly detection. Because of the close association between carotid artery disease and CVD, routine monitoring using our method has the potential to effectively predict CVD onset at an early stage. Key features of the device include cost-effectiveness, portability, and ease of use. Future work will begin with performance analysis of the system using an ultrasound simulation program (e.g. Field II). A hardware prototype will then be built and evaluated using medical-grade custom ultrasound transducers and tissue phantoms. The proposed system will also eventually be modified to create a universal wearable ultrasound device that can monitor and detect plaque deposition in other vulnerable blood vessels, such as the coronary, peripheral, and renal arteries. Such a device is likely to have a significant impact on public health by providing cost-effective early detection of atherosclerosis.

\section{REFERENCES}

[1] World Health Organization, "Cardiovascular diseases (CVDs) fact sheet", http://www.who.int/mediacentre/factsheets/fs317/en/.

[2] J. Habetha, "The MyHeart project-fighting cardiovascular diseases by prevention and early diagnosis", in Proc. of IEEE EMBS Conf., (2006).

[3] T.J. Smilde et. al, "Effect of aggressive versus conventional lipid lowering on atherosclerosis progression in familial hypercholesterolemia (ASAP): a prospective, randomized, double-blind trial", The Lancet, 357.9256 (2001).

[4] J. F. Polak et. al, "The value of carotid artery plaque and intimamedia thickness for incident cardiovascular disease: the multi-ethnic study of atherosclerosis", Journal of the American Heart Association, $2.2(2013)$

[5] V. Nambi et. al, "Carotid intima-media thickness and presence or absence of plaque improves prediction of coronary heart disease risk: the ARIC (Atherosclerosis Risk In Communities) study", Journal of the American College of Cardiology, 55.15 (2010).

[6] K. Nasir et. al, "Heart disease and stroke statistics-2015 update", American Heart Association, (2014).

[7] J. A. Gift, "Carotid collar: a device for auscultory detection of carotid artery stenosis.", Ph.D. thesis, Massachusetts Inst. of Tech. (2003).

[8] E. Awad, "Design of a wearable ultrasound Doppler sensor to monitor blood flow in the common carotid artery", Ph.D. thesis, Massachusetts Inst. of Tech. (1999).

[9] C. Song et. al, "Carotid Plaque Characteristics Detected with High Resolution Nuclear Magnetic Resonance Imaging in Patients with Coronary Artery Disease", Journal of the American College of Cardiology, (2015).

[10] R. Sharma et. al, "A segmentation method for carotid artery atherosclerosis plaque for MRI contrast and MRI features, oxidative stress markers in coronary and carotid plaque", in Proc. of IEEE CBMS Conf., (2003).

[11] T. Nezu et. al, "Carotid intima-media thickness for atherosclerosis", Journal of Atherosclerosis and Thrombosis, 23.1 (2016).

[12] B. Angelsen et. al, "Which transducer array is best?", European Journal of Ultrasound, 2.2 (1995).

[13] Khuri-Yakub, T. Butrus, and O. Oralkan, "Capacitive micromachined ultrasonic transducers for medical imaging and therapy", Journal of Micromechanics and Microengineering, 21.5 (2011).

[14] P.-J. Touboul et. al, "Mannheim carotid intima-media thickness and plaque consensus (2004-2006-2011)", Cerebrovascular Diseases, 34.4 (2012).

[15] Q. Li et. al, "An improved approach for accurate and efficient measurement of common carotid artery intima-media thickness in ultrasound images", BioMed Research International, (2014).

[16] Y. R. Limbu et. al, "Assessment of carotid artery dimensions by ultrasound in non-smoker healthy adults of both sexes", Nepal Medical College Journal: NMCJ, 8.3 (2006).

[17] T. Z. Naqvi and M. S. Lee. "Carotid intima-media thickness and plaque in cardiovascular risk assessment", JACC: Cardiovascular Imaging, 7.10 (2014). 